\title{
Arzt-Entwertung im Internet
}

\section{Otmar Meienberg}

Prof. Dr. med., Facharzt für Neurologie, Mitglied FMH

Ein Praxisbeispiel zeigt wie schwierig es ist, ungerechtfertigte, negative Beurteilungen über eine Ärztin, einen Arzt bei Google löschen zu lassen. Gegen die Anzeige von Arztpraxen auf Google Maps ist nichts einzuwenden. Subjektive Ärzterezensionen von Patienten sind dort aber fehl am Platz, da es immer auch objektive medizinische Gründe dafür geben kann, dass sich Arzt und Patient uneinig sind und der Arzt zudem ans Berufsgeheimnis gebunden ist. Konsequenterweise sollte Google Ärzterezensionen künftig nicht mehr zulassen.

Im April 2016 kam in der TV-Sendung «Puls» von SRF das Thema "Ärztebewertungsplattformen» zur Sprache [1]. Damals hatten Patienten die Wahl zwischen vier Schweizer Plattformen. Es wurde darauf hingewiesen, dass laut den allgemein gültigen Vorgaben des Datenschützers Kommentare nicht ehrverletzend sein dürfen. Zur Erfüllung dieser Auflage hatten alle vier Bewertungsplattformen unterschiedliche, aber angemessene Massnahmen getroffen.

Kritisch wurde in der Sendung vermerkt, dass damals die Zahl der Nutzer für eine angemessene Qualitätsbeurteilung noch zu gering war und dass viele Ärzte bisher bloss einzelne oder gar keine Beurteilungen erhalten hatten.

Hat sich bis heute diesbezüglich etwas geändert? Die Zahl der Nutzer hat zugenommen. Wirklich brauchbare und für Patienten, welche einen geeigneten Arzt suchen hilfreiche Bewertungen sind weiterhin rar. Ein

\section{Resumé}

Un exemple pratique montre à quel point il est difficile de faire supprimer de Google une évaluation négative injustifiée d'un médecin. II n'y a rien à redire à la localisation des cabinets médicaux sur Google Maps. Les avis subjectifs des patients sur les médecins n'y ont toutefois pas leur place, car des raisons médicales objectives peuvent expliquer les désaccords entre patient et médecin, ce dernier étant en outre tenu au secret professionnel. Il faut par conséquent trouver des moyens d'empêcher les évaluations des médecins sur Google à l'avenir. wesentlicher Grund dafür: mit ihrem Arzt zufriedene Patienten, im Gegensatz zu unzufriedenen, sehen sich kaum veranlasst, dies öffentlich im Internet kundzutun. Durch stark subjektiv gefärbte negative Bewertungen können so selbst von besten Ärzten Zerrbilder entstehen.

Diese Probleme versucht das Ärzteverzeichnis Schweiz «doktor.ch» [2] zu vermeiden. Unter «Bewertungen» ist dort vermerkt: «Google ist nicht nur die meistgebrauchte, sondern auch die wichtigste Bewertungsplattform im Schweizer Internet. Wir verlinken direkt zu den Google-Suchresultaten. Die Bewertungen werden von Google kontrolliert.» Ergänzend wird darauf hingewiesen, dass auch falsche oder manipulierte Bewertungen bei Google veröffentlicht sein können und deshalb die Bewertungen (= Rezensionen) immer auch kritisch beurteilt werden müssten.

\section{Schlechte Bewertungen bei Google - ein Erfahrungsbericht}

Lange auf eine solche Google-Bewertung zu warten brauchte ich nicht: Jemand Wohlwollender machte mich darauf aufmerksam, dass bei der Google-Suche «neurologie am schaulager» überlagert über die gesuchten Informationen ein unübersehbares Fenster mit drei Google-Rezensionen erscheine (Abb.1).

Von den drei Rezensionen mit den schlechtestmöglichen Bewertungen betraf eine mich, die andern beiden zwei meiner Praxiskollegen. Die Botschaft im abgebildeten Fenster war klar: In eine Praxis mit gleich drei miserablen arroganten Ärzten will keiner! Hilfreich bot Google unten im selben Fenster mit "Andere suchen auch nach ...» als Alternativen 
eine Reihe von zumeist nicht bewerteten Neurologen an. - Ein Globalangriff auf unsere Praxisgemeinschaft: Durch diese «Sammel»-Rezension wurden gleich drei Ärzte auf einen Streich getroffen! Dies ohne Berücksichtigung der Tatsache, dass auch in einer Praxisgemeinschaft jeder Einzelne voll ausgebildete Facharzt für seine ärztliche Tätigkeit alleine verantwortlich ist. Der Gruppenangriff erinnert mich ans Märchen vom "tapferen Schneiderlein",

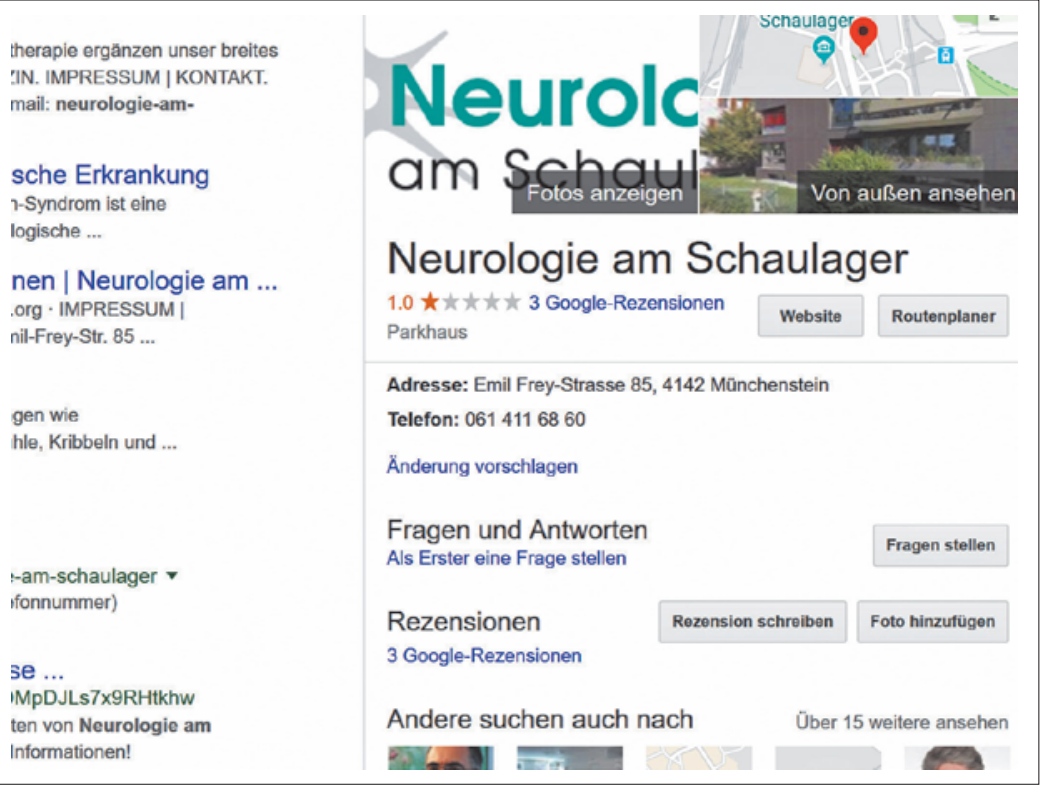

Abbildung 1: Drei negative Google-Sammel-Rezensionen von 3 verschiedenen Neurologen zu unserer Praxis und Empfehlung anderer Neurologen.

\section{Neurologie am Schaulager}

Emil Frey-Strasse 85, Münchenstein

$$
1.0 \star \star \star \star \star 3 \text { Rezensionen }
$$$$
\text { It Hilfreich? }
$$$$
\text { R Roro }
$$

Roro
1 Rezension

$\star \star \star \star \star \star$ vor 3 Wochen

(Ubersetzt von Google) Sah Dr. Meienberg. Er war arrogant, hörte mir nicht zu. Ich habe mir nur die Dokumente angesehen, die ich mitgebracht habe, aber das hat nicht das ganze Bild gemalt, und das Doker waren zwei Jahre at. cher $P$ sychologen in Geh da nicht hin. Es gibt einen Grund, warum sie dich so schnell nehmen können!

\section{(Original)}

Saw Dr Meienberg. He was arrogant, didn't listen to me. Just looked at the documents that I had brought but that did not paint the whole picture and that were 2 years old. He was not intrested in what i had to say. He made his mind up very quickly that my problem was psychological even though he did not talk to me and I am followed by a psychologist who agrees that it is not. But no that has no value because he is not a psychiatrist! Sent me away saying I should go to a Klink. Do not go there. There is a reason they can take you so fast mit dem Unterschied, dass es keinen Mut braucht, anonym und klammheimlich zu agieren, ohne die öffentlich im Internet Angegriffenen auf die Tat hinzuweisen. - Dass eine angegriffene und verletzte Wildsau einmal auch einen Urheber wittern, und zum Gegenangriff übergehen könnte, damit hat man nicht gerechnet.

Die für sie kostenlose anonyme Rezension meiner ärztlichen Tätigkeit durch die Patientin mit Pseudonym «Roro» (Abb. 2) ist in verschiedenerlei Hinsicht beachtenswert:

Die Übersetzung ihres englischen Originaltextes auf Deutsch ist sprachlich miserabel und dadurch inhaltlich teils missverständlich. Dies, und vor allem potentiell rufschädigende Äusserungen liessen bei mir Zweifel aufkommen, ob ihre Bewertung von Google kontrolliert wurde, wie von der Such- und Bewertungsplattform "doktor.ch" behauptet, oder ob eine externe Manipulation vorliege, auf welche dort hingewiesen wurde. - Vor allem aber waren die Vorwürfe der Patientin masslos übertrieben und teils ehrverletzend. Bei der Google-Suche nach einer Möglichkeit, die unzutreffende negative Bewertung zu entfernen, bin ich mit Phrasen wie: «arztbewertung löschen» auf zahllose Angebote gestossen, dies gegen Bezahlung für mich zu erledigen. Nur bei Google selber bin ich trotz intensiver Suche nicht fündig geworden. («Änderung vorschlagen" [siehe Abb. 1] betrifft nur die Zugangsdaten zur Praxis, nicht jedoch die Rezensionen ...) Schliesslich stiess ich auf eine Homepage, deren Design Google ähnlich sah [3]. Unter «Bewertung löschen» zeigte sich folgendes Bild (Abb. 3): Ein teures Angebot für umgerechnet 209 CHF mit nur geringen Erfolgsaussichten! Im «Shop (Abb. 4)» kann man sich zudem positive Bewertungen mit Mengenrabatten kaufen ...

Da mir keiner der Vorschläge sympathisch war, habe ich mich von einem Juristen meiner Ärzte-Rechtsschutz-Versicherung beraten lassen und meiner Patientin einen eingeschriebenen Brief geschickt mit einer Kopie ihrer Rezension und einer Kopie meines Untersuchungsberichtes an ihre Hausärztin zum Vergleich der medizinischen Tatsachen mit ihren diversen unzutreffenden Behauptungen. Zudem fügte ich eine Rechtsbelehrung bezüglich Ehrverletzungen bei und teilte ihr mit, dass ich noch abklären liesse, ob ihre Rezension den Tatbestand einer üblen Nachrede oder gar einer Verleumdung erfülle. Als Vorschlag zur Güte schlug ich ihr vor, dass sie ihre Google-Bewertung umgehend löschen lasse. - Zwei Tage, nachdem sie meinen Brief erhalten hatte, war ihre Bewertung gelöscht (Abb. 5).

Die Rezension von «Roro» (s. Abb. 2) - zuvor unterhalb von «tobelerhone» - fehlt nun. Die negativen Rezensio- 


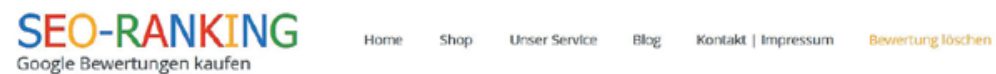

\section{Google Bewertung löschen}
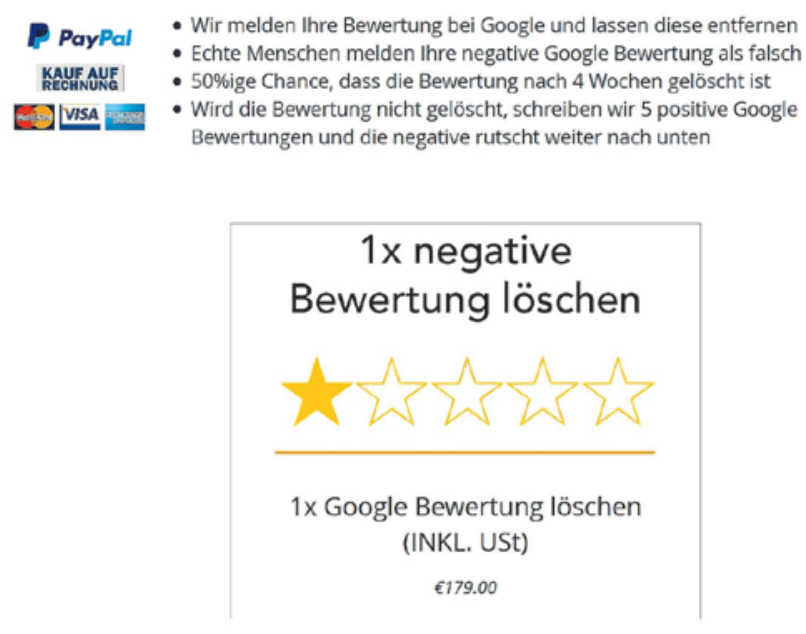

\section{oder zum Shop: \\ positive Google Bewertungen kaufen}

Abbildung 3: Zweifelhaftes Angebot im Internet (https://www.googlebewertungen. com), unliebsame negative Google-Bewertungen zu löschen.

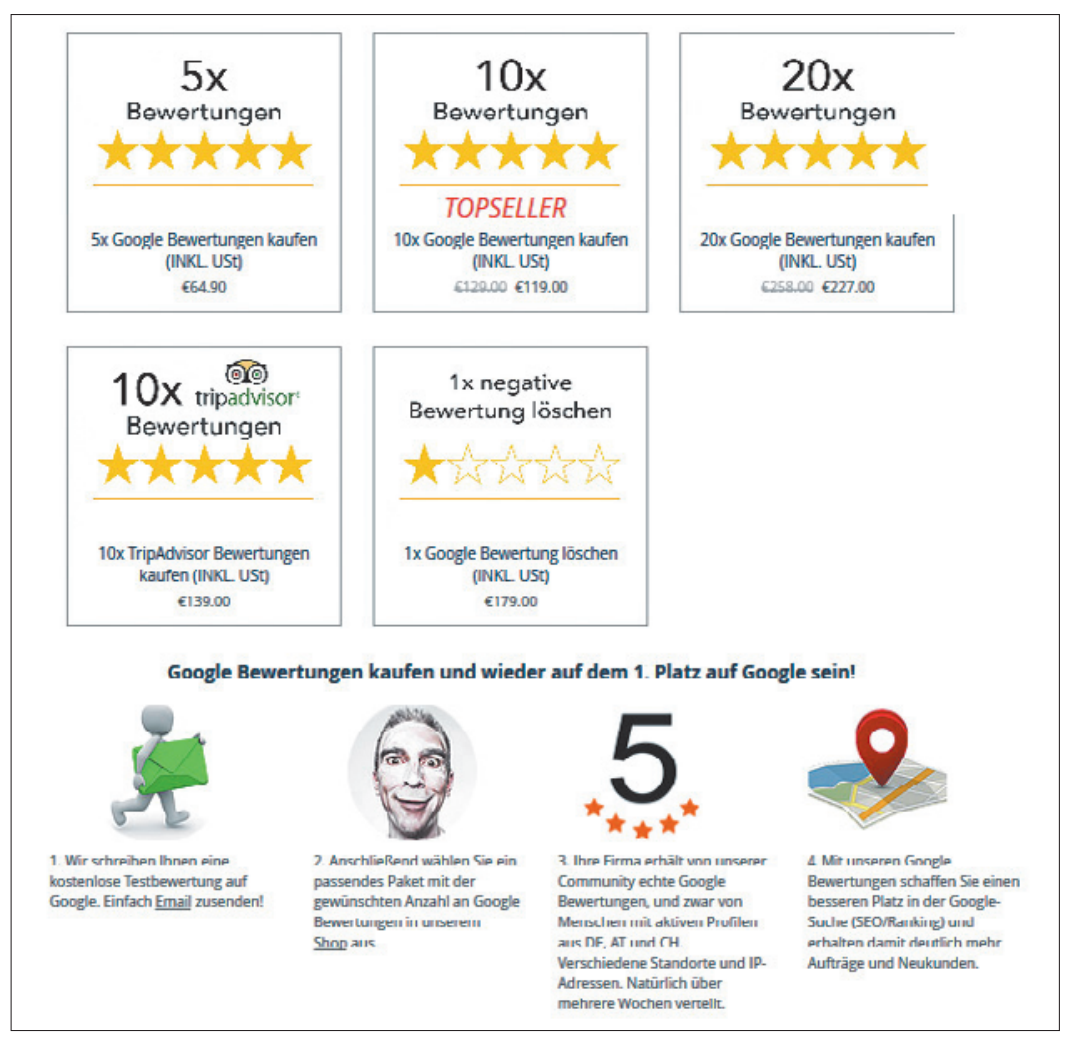

Abbildung 4: Angebote im Internet (https://www.googlebewertungen.com): sich mit konstruierten «Fake»-Bewertungen an die Spitze katapultieren zu lassen. nen meiner beiden Praxiskollegen sind geblieben - die eine bereits seit 6 , die andere seit 3 Monaten - voraussichtlich aber für immer, wenn sie dagegen nichts unternehmen. Oft weiss man aber nicht, wer die anonymen Rezensoren sind oder man hat keine Kenntnis von einer Rezension. Müssen wir Ärzte nun täglich im Internet nach allfälligen negativen Rezensionen suchen? - Neu sind zudem in Abb. 5 ein maximal negatives (Fifi Brin, 1 Stern) und ein maximal positives (Fiercely Girl, 5 Sterne) fingiertes «fake rating», um zu zeigen, wie sich solche auf die Gesamtbewertung auswirken können, und wie durch unten im Fenster angefügte hinzugekaufte positive Ratings (Abb. 4) unerwünschte Rezensionen oben im Fenster zum Verschwinden zu bringen sind.

\section{Weltweite Verbreitung negativer Ärzte-Rezensionen mit «Google Maps»}

Sensibilisiert durch obige Erfahrungen halte ich ab und zu Ausschau nach weiteren Google-Rezensionen von unserer Gemeinschaftspraxis und mir.

Dabei bin ich auf ein weiteres Phänomen gestossen: Ein mit mir offenbar zufriedener Patient hat mich mit dem Maximum an verfügbaren 5 Sternen belohnt. Wow! Bei näherem Hinsehen bezeichnete er sich als «Local Guide» Level 6 und wies bisher 84 Rezensionen aus. Diese betrafen Pizza-Kuriere, Shopping Centers, Autowaschanlagen, eine Waldschule, Sportgeschäfte, Moto Shops etc. Vorläufig war ich der erste Arzt, dem von ihm die Ehre widerfuhr, in diese illustre Gesellschaft aufgenommen zu werden. - Da ich mir aber etwas deplatziert vorkam, googelte ich mit der Frage: Was sind «Local Guides»?. Folgender Link schaffte für mich Klarheit: https://www.turn-on.de/tech/ratgeber/ google-local-guides-das-steckt-dahinter-310454 Google will seine weltweit zugänglichen Maps für die Nutzer mit möglichst vielen hilfreichen Informationen über Orte, Restaurants, Attraktionen etc. versorgen. Als «Local Guide» kann man mit derartigen Angaben Punkte sammeln und sich bis zu einem Level von 10 hocharbeiten mit der Aussicht, als Fremdenführer Geld zu verdienen. Das Dumme dabei ist bloss, dass auf den Google Maps dann auch weltweit inadaequate negative Ärzterezensionen zu finden sind!

Gegen die Anzeige von Arztpraxen auf Google Maps ist nichts einzuwenden. Subjektive Ärzterezensionen von Patienten sind dort aber fehl am Platz, da es immer auch objektive medizinische Gründe dafür geben kann, dass sich Arzt und Patient uneinig sind und der Arzt zudem ans Berufsgeheimnis gebunden ist. Subjektive negative Arztrezensionen erfolgen oft nach «Local Guide»-Kriterien: Eine lange Wartezeit bei einer 


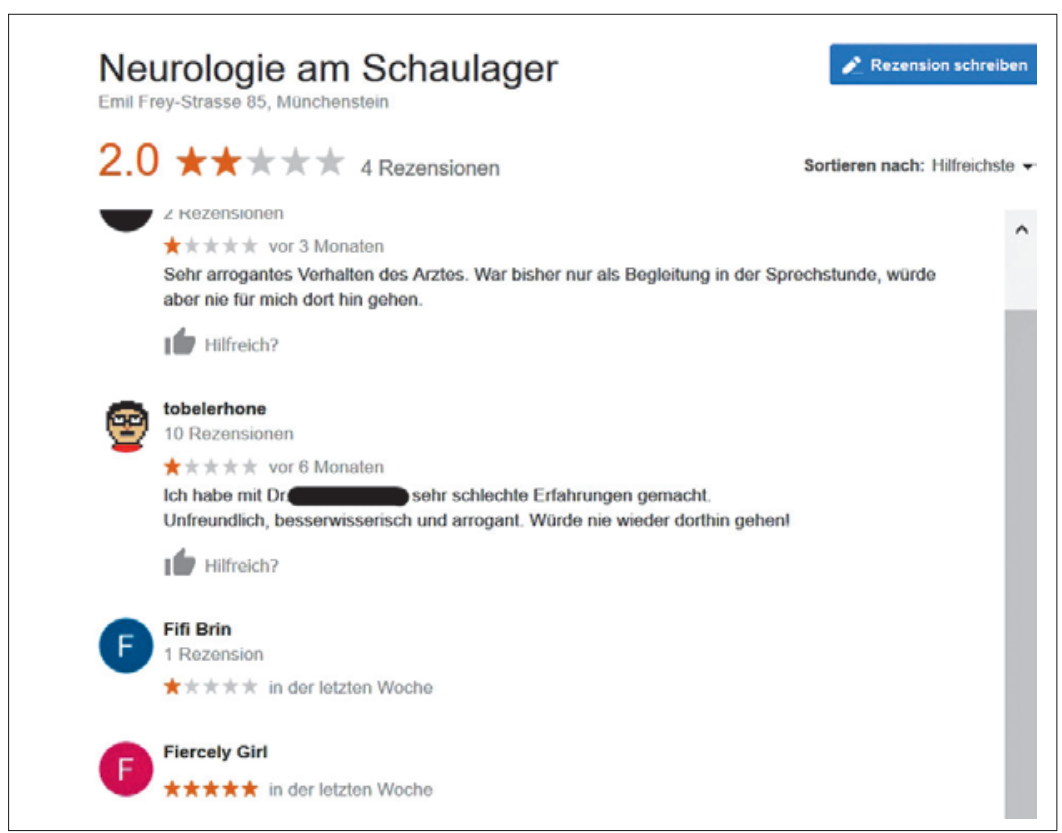

Abbildung 5: Von «Roro» (vgl. Abb. 2) gelöschte Google-Rezension 2 Tage nach Erhalt meines eingeschriebenen Briefes. Zudem: Aufwertung unserer Praxis (vgl. Abb.1) von 1 Stern (= miserabel) auf 2 Sterne und Demonstration, wie sich gekaufte fingierte («Fake») Bewertungen (Fiercely Girl maximal positiv) auf die Praxisgesamtbewertung auswirken.
Korrespondenz:

Prof. Dr. med.

Otmar Meienberg otmar.meienberg[at]hin.ch www.neurologie-amschaulager.ch
Autowaschanlage mag berechtigterweise Anlass zu einer Beschwerde geben, nicht aber in einer Arztpraxis, wenn der Hausarzt einen Notfall hat oder der Fachspezialist vom Patienten eben erst mitgebrachte umfangreiche komplexe Akten studieren muss.

\section{Fazit}

1 Ärzteratings gehören nicht auf weltweit zugängliche Google Maps.

2 Angesichts der kommerziellen Angebote von «Fake»-Bewertungen sind Ärzte-Ratings im Internet wertlos geworden. (Schlimmstenfalls kann jemand anonym gar einen Arzt negativ bewerten, ohne bei ihm je Patient gewesen zu sein.)

Einzig richtige Konsequenz wäre es, Google-Ratings von Ärzten im Internet abzuschaffen!

Für Google wäre es ein Leichtes, im Rating-Eingabefenster den Vermerk anzubringen: «Keine Ärzte-Rezensionen!»

Dafür gibt es relevante objektive Gründe:

- Gegen konsumoptimierende oder tourismusfördernde Rezensionen durch «Local Guides» ist nichts einzuwenden. Arztrezensionen sind aber hier, wie auch auf «Google Maps» fehl am Platz!

- Gute, von Patienten naturgemäss immer erwartete ärztliche Leistungen werden kaum je unaufgefordert in der Öffentlichkeit mit positiven Google-Rezensionen «belohnt».

- Anlass zu negativen subjektiven Rezensionen geben oft Beurteilungen der Arbeitsfähigkeit, Autofahrtauglichkeit, Rentenansprüche etc. und stammen nicht selten von Patienten mit psychischen Hintergrundsproblemen, alles Dinge, gegen welche man sich wegen des Arztgeheimnisses in der Öffentlichkeit nicht zur Wehr setzen kann!

Ich bin mir bewusst, dass es mir mit diesem Artikel allein nicht gelingen wird, Google zur Abschaffung von Ärzte-Ratings zu bewegen. Wäre es da nicht Aufgabe unserer Standesorganisation, sich mit dem die gesamte Ärzteschaft betreffenden Anliegen an Google zu wenden, möglichst nach vorherigem Einholen der Unterstützung des eidgenössischen Datenschützers (s. den ersten Abschnitt dieses Artikels)?

Bei Missständen, wie hier aufgezeigt, hat Google immerhin zu bedenken, dass die Glaubhaftigkeit und Verlässlichkeit seiner sämtlichen Rezensionen auf dem Spiel steht, und die Gefahr droht, dass sie künftig von niemandem mehr ernst genommen werden!

Mit dem Ausschluss von Ärzte-Rezensionen könnte Google ein Zeichen setzen, dass relevante Anliegen seiner Nutzer ernst genommen werden.

Bis handfeste korrigierende Massnahmen beantragt und allenfalls von Google auch umgesetzt werden, dürfte noch einige Zeit vergehen.

Mein Anliegen ist es deshalb, mit diesem Artikel die praxistätige Ärzteschaft schon jetzt auf die Missstände warnend hinzuweisen und Tipps zu geben, wie man sich nötigenfalls effizient zur Wehr setzen kann (s. Beispiel Patientin «Roro»). Einem Kollegen von mir hat diese Methode ebenfalls umgehend geholfen.

\section{Literatur}

1 Büchi S. Arztbewertung - Wie viele Sterne dürfen's denn sein? SRF TV-Sendung «Puls» vom 18.04.2016 (aktualisiert 20.04.16). Video und ergänzender Übersichtstext. https://www.srf.ch/sendungen/ puls/gesundheitswesen/arztbewertung-wie-viele-sterne-duerfens-denn-sein

2 Inforating, Ärzteverzeichnis Schweiz «Doktor.ch»: http://www.doktor.ch

3 Negative Bewertung löschen - positive Bewertungen kaufen: https://www.googlebewertungen.com 